\title{
Efeito de Trichoderma spp. no crescimento de mudas clonais de Eucalyptus camaldulensis
}

\author{
Effect of Trichoderma spp. on Eucalyptus \\ camaldulensis clonal seedlings growth
}

\author{
Gileno Brito de Azevedo', Quelmo Silva de Novaes², Glauce Taís de Oliveira Sousa Azevedo1, \\ Helane França Silva ${ }^{3}$, Geraldo Gomes Rocha Sobrinho ${ }^{4}$ e Adalberto Brito de Novaes ${ }^{2}$
}

\begin{abstract}
Resumo
Fungos do gênero Trichoderma são largamente utilizados no biocontrole de fitopatógenos, além de serem capazes de estabelecer associações com plantas, aumentando seu potencial de desenvolvimento. Este trabalho avaliou o desenvolvimento e qualidade de mudas clonais de Eucalyptus camaldulensis (clone 58) inoculadas com duas espécies de Trichoderma: T. harzianum e T. virens, por diferentes métodos, a saber: tratamento das miniestacas; incorporação de grãos de arroz colonizados ao substrato; pulverização de suspensão de esporos e; adição de suspensão de esporos ao substrato + pulverização de suspensão de esporos. O uso de Trichoderma proporcionou melhor desempenho para todas as variáveis analisadas, sendo os maiores incrementos verificados no número de folhas $(110 \%)$, massas secas aérea $(70,4 \%)$, das raízes $(54,7 \%)$ e total $(64,8 \%)$ e índice de qualidade de Dickson $(70,4 \%)$, em relação à testemunha, sem Trichoderma. O tratamento das minestacas com $T$. virens mostrou ser o tratamento mais eficiente na promoção do crescimento e qualidade das mudas. As duas espécies de Trichoderma foram detectadas nas raízes pelo teste de colonização endofítica. Seu uso mostrou ser uma alternativa promissora a ser adotada nos viveiros florestais, pois, além de atuar no biocontrole de fitopatógenos, foi capaz de promover o desenvolvimento das mudas.
\end{abstract}

Palavras-chave: Miniestaquia, promoção do crescimento, eucalipto, Trichoderma virens, Trichoderma harzianum.

\begin{abstract}
Fungi of the genus Trichoderma are widely used in biological control of phytological pathogens, besides being able to establish associations with plants, increasing their development potential. This work evaluated the development and quality of clonal seedlings of Eucalyptus camaldulensis (clone 58) inoculated with two species of Trichoderma: T. harzianum and T. virens, by different methods: treatment of mini-cuttings; incorporating grains of rice colonized in the substrate; spraying of spore suspension and adding spore suspension to the substrate + spraying of spore suspension. The use of Trichoderma provides better performance for all variables, with the largest increases observed in the number of leaves $(110 \%)$, dry mass of shoot $(70.4 \%)$, roots $(54.7 \%)$ and total $(64,8 \%)$ and Dickson quality index $(70.4 \%)$ compared to the control, without Trichoderma. Treatment of mini-cutting with $T$. virens tends to be the most effective treatment in promoting the growth and quality of seedlings. The two species of Trichoderma were found in the roots by endophytic colonization test. Its use proved to be a promising alternative to be adopted in forest nurseries, because in addition to acting in the biological control of plant pathogens, it was able to promote the development of eucalyptus seedlings in nursery conditions.
\end{abstract}

Keywords: Mini-cutting, growth promotion, eucalyptus, Trichoderma virens, Trichoderma harzianum.

${ }^{1}$ Professor Assistente. UFMS -Universidade Federal de Mato Grosso do Sul, Campus de Chapadão do Sul. Rodovia MS-306 - Zona Rural, Chapadão do Sul - MS, Brasil. CEP 79560-000. E-mail: gileno.azevedo@ufms.br; glauce.azevedo@ufms.br 2Professor Titular do Departamento de Fitotecnia e Zootecnia. UESB - Universidade Estadual do Sudoeste da Bahia. Estrada do Bem Querer - Km 04 - Caixa-postal: 95 - 45031-300 - Vitória da Conquista, BA, Brasil. E-mail: qsnovaes@uesb.edu. br; adalberto.brito@globo.com

3Professora Assistente. UFPI-Universidade Federal do Piauí, Campus Professora Cinobelina Elvas. Rodovia Municipal Bom Jesus - Viana, km 01 Planalto Horizonte. Bom Jesus, PI, Brasil CEP 64900-000. E-mail: helane.engflo@gmail.com

${ }^{4}$ Doutorando em Agronomia. UESB - Universidade Estadual do Sudoeste da Bahia. Estrada do Bem Querer - Km 04 Caixa-postal: 95 - 45031-300 - Vitoria da Conquista, BA, Brasil. E-mail: ggomesrocha@hotmail.com

Sci. For., Piracicaba, v. 45, n. 114, p. 343-352, jun. 2017 DOI: dx.doi.org/10.18671/scifor.v45n114.10 


\section{INTRODUÇÃO}

As espécies do gênero Eucalyptus, devido às suas características de adaptação às diferentes condições de clima e solo e diversificação do uso de seus produtos, têm sido amplamente utilizadas em plantios florestais, contribuindo para diminuir a pressão sobre as florestas nativas. No Brasil, segundo informações do Serviço Florestal Brasileiro (SFB, 2015), no ano de 2014, a área de florestas plantadas ultrapassou 9,3 milhões de hectares, com destaque para as espécies desse gênero, as quais representam $74,23 \%$ desse total.

Dentre outros fatores, o êxito da formação de florestas de alta produtividade depende em grande parte do padrão de qualidade das mudas utilizadas (GOMES; PAIVA, 2004). Ainda que as técnicas de produção de mudas florestais sejam bem consolidadas, há uma busca constante por alternativas de manejo que estimulem o desenvolvimento das mudas, permitindo reduzir o tempo necessário para sua formação e garantindo qualidade superior. Uma alternativa é o uso de microrganismos, como os fungos do gênero Trichoderma, no processo de produção de mudas (CARVALHO FILHO et al., 2008a; CHÁVEZ et al., 2014; FORTES et al., 2007; MACHADO et al., 2015).

O gênero Trichoderma compreende um grande número de espécies de fungos filamentosos de vida livre, encontrados em vários ecossistemas, habitando desde as regiões tropicais até as temperadas (BROTMAN et al., 2010; MACHADO et al., 2012). São comumente encontrados nos solos, associado às raízes das plantas (BENÍTEZ et al., 2004; HARMAN et al., 2004) e na decomposição de restos vegetais e madeira, sendo raramente associados a doenças de plantas (SRIVASTAVA et al., 2014). Trichoderma se destaca pela alta capacidade reprodutiva, sobrevivência em condições adversas, eficiência na utilização de nutrientes, capacidade de modificar a rizosfera e ação antagônica a fungos fitopatogênicos (BENÍTEZ et al., 2004).

O Trichoderma é largamente utilizado no biocontrole de alguns fitopatógenos de importância econômica em todo o mundo (HARMAN, 2000; 2006; SABA et al., 2012; SILVA et al., 2014; SRIVASTAVA et al., 2014; ZALDÚA; SANFUENTES, 2010;), uma vez que possuem propriedades antagônicas a estes microrganismos, podendo reduzir sua ação através da competição por nutrientes e espaço, micoparasitismo e antibiose (BENÍTEZ et al., 2004; BROTMAN et al., 2010; HARMAN, 2006; HERMOSA et al., 2012). Além disso, estudos têm revelado que fungos do gênero Trichoderma podem interagir diretamente com as raízes ou outras partes das plantas (colonização endofítica), aumentando o potencial de crescimento (HERMOSA et al., 2012), a resistência às doenças e a tolerância à estresses bióticos e abióticos das plantas (BROTMAN et al., 2013; HERMOSA et al., 2012; SRIVASTAVA et al., 2014).

Inicialmente, a promoção de crescimento de plantas pela aplicação de Trichoderma foi relacionada ao controle dos microrganismos prejudiciais presentes no solo (MACHADO et al., 2012). Porém, alguns autores apontam que a associação do Trichoderma com as raízes promove o crescimento das mesmas através da produção de fitohormônios e aumento da disponibilidade e maior eficiência no uso de alguns nutrientes pelas plantas (AZARMI et al., 2011; BROTMAN et al., 2010; GRAVEL et al., 2007; STEWART; HILL, 2014). O efeito benéfico do Trichoderma tem sido verificado no crescimento e desenvolvimento de várias culturas, como soja (HARMAN, 2000), milho (HARMAN, 2000; RESENDE et al., 2004), feijão (CHAGAS JÚNIOR et al., 2014; PEDRO et al., 2012), algodão (SHANMUGAIAH et al., 2009), cana-de-açúcar (SINGH et al., 2010), maracujazeiro (SANTOS et al., 2010), tomate (AZARMI et al., 2011), pimentão (HARMAN, 2000) e pepino (SILVA et al., 2011).

Contudo, ainda são poucos os trabalhos que investigaram o uso de Trichoderma na promoção do crescimento de espécies florestais. Estudos com o híbrido urograndis (CARVALHO FILHO et al., 2008a; SANTOS et al., 2008), Eucalyptus sp. (FORTES et al., 2007), Pinus radiata (CHÁVEZ et al., 2014; DONOSO et al., 2008), Gochnatia polymorpha (MACHADO et al., 2015) e Hevea brasiliensis (PROMWEE et al., 2014), apresentaram resultados promissores. No entanto, segundo Benítez et al. (2004), o mecanismo de ação do Trichoderma pode variar de acordo com a espécie, o tipo de cultivo e as condições ambientais (disponibilidade de nutrientes, $\mathrm{pH}$ ou temperatura). A esses fatores, também pode ser incluído a forma de inoculação do Trichoderma, que não é padronizada entre os estudos.

Nesse contexto, o presente trabalho teve como objetivo avaliar o efeito de duas espécies de Trichoderma: T. virens e T. harzianum, inoculadas por diferentes métodos, no crescimento e qualidade de mudas clonais de Eucalyptus camaldulensis. 


\section{MATERIAL E MÉTODOS}

\section{Local de estudo}

O experimento foi conduzido no período de janeiro a abril de 2011, no viveiro comercial da Tecnoverde, localizado nas coordenadas $14^{\circ} 55^{\prime} \mathrm{S}$ e $40^{\circ} 53^{\prime} \mathrm{W}$, a uma altitude de $871 \mathrm{~m}$, no município de Vitória da Conquista-BA. De acordo com a classificação de Köppen, a região apresenta clima tropical de altitude Cwb e as médias de temperaturas mínimas e máximas são de $16,1^{\circ} \mathrm{C}$ e $25,3^{\circ} \mathrm{C}$, respectivamente. A precipitação média anual é de $733,9 \mathrm{~mm}$, distribuída principalmente nos meses de novembro a março (SEI, 2010).

\section{Sistema de produção das mudas}

O material genético utilizado na produção das mudas foi o clone 58 de Eucalyptus camaldulensis Dehnh., registrado RNC/MAPA $\mathrm{n}^{\circ}$ 21394. As mudas foram produzidas através da técnica de miniestaquia, sendo utilizadas miniestacas apicais oriundas do minijardim clonal do tipo semi-hidropônico (canaletão), com tamanho variando de 7 a $10 \mathrm{~cm}$ e três pares de folhas estendidas, que tiveram $50 \%$ da área foliar reduzida através do corte do limbo foliar.

Para a produção das mudas foram usados recipientes plásticos, do tipo tubetes, com capacidade de $55 \mathrm{~cm}^{3}$ previamente esterilizados, os quais foram preenchidos com substrato a base de casca de pinus, vermiculita e casca de arroz carbonizada, na proporção 1:1:1 sendo adicionado, para cada litro de substrato, $3 \mathrm{~g}$ do adubo de liberação lenta Osmocote 15-10-10 + micronutrientes (15\% de $\mathrm{N} ; 10 \%$ de $\mathrm{P}_{2} \mathrm{O}_{5}$ e $10 \%$ de $\mathrm{K}_{2} \mathrm{O} ; 3,5 \%$ de Ca; $1,5 \%$ de $\mathrm{Mg} ; 3,0 \%$ de $\mathrm{S} ; 0,02 \%$ de $\mathrm{B} ; 0,05 \%$ de $\mathrm{Cu} ; 0,5 \%$ de Fe; $0,1 \%$ de $\mathrm{Mn} ; 0,004 \%$ de Mo e 0,05\% de Zn). As miniestacas foram plantadas no substrato, e colocadas para enraizar em casa de vegetação, sendo a irrigação realizada por meio de nebulização automatizada, onde permaneceram até 30 dias após o estaqueamento (DAE). Após esse período, as mudas foram transferidas para área com sombreamento de $50 \%$ por um período de 10 dias e passaram a ser irrigadas por microaspersão. Posteriormente, foram transferidas para canteiros suspensos a pleno sol por um período de 40 dias. Em seguida, foram transferidas para área de pleno sol, até completarem os 95 DAE.

\section{Obtenção e preparo dos inóculos de Trichoderma}

Neste experimento foram utilizadas duas espécies de Trichoderma: T. virens e T. harzianum, cedidas pela Empresa BIOFUNGI - Controle Biológico. Para a obtenção dos inóculos, as espécies foram multiplicadas em grãos de arroz no Laboratório de Fitopatologia da Universidade Estadual do Sudoeste da Bahia. Para tanto, foi realizado o pré-cozimento do arroz em água quente por 10 minutos, sendo posteriormente transferido para sacos de polipropileno, na proporção de $300 \mathrm{~g}$ de arroz/ saco, e autoclavado a $121^{\circ} \mathrm{C}$ durante 20 minutos. Em seguida, as espécies de Trichoderma, separadamente, foram inoculadas no arroz e, posteriormente, acondicionados em câmara de germinação do tipo Biochemical Oxygen Demand (B.O.D.) à temperatura de $\pm 25^{\circ} \mathrm{C}$ e fotoperíodo de 12 horas, por um período de sete dias. Diariamente, os sacos contendo o arroz foram revolvidos para facilitar as trocas gasosas, quebra dos micélios e aumento da esporulação. Sete dias após a inoculação, o arroz colonizado foi armazenado em geladeira até a instalação do experimento no viveiro.

\section{Métodos de inoculação de Trichoderma}

A fim de se verificar se a forma de inoculação interfere no desempenho do Trichoderma, as inoculações foram realizadas por diferentes métodos:

a) Tratamento das miniestacas (TM): Durante o estaqueamento no substrato, as miniestacas tiveram a base imersa em pó de arroz colonizado pelas espécies de Trichoderma. O pó foi obtido a partir da trituração dos grãos de arroz colonizados em liquidificador doméstico durante cinco minutos.

b) Incorporação de grãos de arroz colonizados ao substrato (IGS): Antes do preenchimento dos tubetes, o arroz colonizado pelo Trichoderma foi incorporado ao substrato de produção das mudas, numa concentração de 1\% (peso/peso).

c) Pulverização de suspensão de esporos (PSE): Foram realizadas três pulverizações foliares com a suspensão de esporos de Trichoderma, sendo a primeira realizada logo após o estaqueamento, e as demais aos 30 e 60 DAE. Durante as aplicações, foram utilizados pulverizadores manuais e fôrmas 
confeccionadas de papelão, a fim de garantir a inoculação do fungo somente na parcela desejada. A suspensão de esporos foi obtida após a lavagem dos grãos de arroz colonizados em água destilada e, com auxílio de uma câmara de Neubauer, foi calibrada uma suspensão fúngica contendo $1 \times 10^{8}$ conídios $\mathrm{mL}^{-1}$.

d) Adição de suspensão de esporos ao substrato + pulverização de suspensão de esporos (ASS+PSE): Antes do preenchimento dos tubetes, foi incorporado ao substrato $100 \mathrm{~mL}$ da suspensão de esporos $\left(1 \times 10^{8}\right.$ conídios $\left.\mathrm{mL}^{-1}\right)$, para cada $30 \mathrm{~kg}$ de substrato. As pulverizações foliares com a suspensão de esporos foram realizadas aos 30 e 60 DAE.

\section{Avaliação do desenvolvimento e qualidade das mudas}

As variáveis morfológicas e suas relações, utilizadas nas avaliações aos 95 DAE foram: altura da parte aérea (H), em centímetros; diâmetro na base do caule (D), em milímetros; número de folhas (NF); massa seca aérea (MSA), das raízes (MSR) e total (MST), em gramas; as relações altura/diâmetro (H/D) e altura/massa seca aérea (H/MSA); e o índice de qualidade de Dickson (IQD = [MST] / [(H/D + MSA/MSR]). A altura da parte aérea foi obtida com auxílio de uma régua, medindo-se desde a base da muda até a gema apical. O diâmetro da base do caule foi medido com auxílio de um paquímetro digital, com precisão de $0,01 \mathrm{~mm}$, na altura do substrato. O número de folhas foi obtido através da contagem direta. Os valores de massa seca foram determinados após a lavagem do sistema radicular para a retirada do substrato, sendo as mudas seccionadas na altura da base do caule, visando separar o sistema radicular da parte aérea, com posterior secagem em estufa a 75 ${ }^{\circ} \mathrm{C}$, até obtenção da massa seca constante, e pesagem em balança analítica, com precisão de 0,001 g (BÖHM, 1979).

O experimento foi delineado em blocos casualizados (DBC), com nove tratamentos dispostos em esquema fatorial $(2 \times 4)+1$, com Trichoderma virens e Trichoderma harzianum e quatro métodos de inoculação (TM, IGS, PSE e ASS+PSE), acrescido do tratamento sem Trichoderma (testemunha), com quatro repetições, cada uma com oito mudas. Após verificados os pressupostos da homogeneidade e normalidade, os dados foram submetidos à análise de variância $(=0,05)$, sendo as médias comparadas estatisticamente através do teste de Tukey $(=0,05)$. As análises foram realizadas com auxílio do pacote ExpDes do software R, versão 3.1.3 (FERREIRA et al., 2014). De forma complementar, a fim de auxiliar na decisão do melhor tratamento, foi avaliada a diferença relativa entre os tratamentos onde se usou Trichoderma e onde não se usou.

\section{Teste de colonização endofítica por Trichoderma}

O teste de colonização endofítica foi realizado a fim de se avaliar a presença de Trichoderma no interior de órgãos e tecidos, como raízes, caules e folhas das mudas de eucalipto. Para tanto, aos 95 DAE, duas mudas de cada tratamento foram lavadas em água e, em seguida, de cada muda foram retirados três fragmentos de $\pm 1 \mathrm{~cm}$ das raízes e do caule e três discos de $7 \mathrm{~mm}$ de diâmetro nas folhas, que passaram por processos de desinfecção superficial, através de imersões em solução de álcool $70 \%$ durante $1 \mathrm{~min}$, hipoclorito de sódio a 2,5\% durante $4 \mathrm{~min}$, solução de álcool 70\% durante 30 seg e duas vezes em água destilada durante $1 \mathrm{~min}$. Em seguida, o material desinfestado foi disposto em placas de Petri de $90 \mathrm{~mm}$ contendo o meio de cultura BDA, as quais foram mantidas em B.O.D. com temperatura de $\pm 25^{\circ} \mathrm{C}$ e fotoperíodo de 12 horas por um período de cinco dias. Após esse período, com auxílio de um microscópio estereoscópico foi verificado se o Trichoderma estava presente $(+)$ ou ausente $(-)$ nas diferentes amostras.

\section{RESULTADOS E DISCUSSÃO}

Na Tabela 1 são apresentadas as respostas do desenvolvimento e qualidade das mudas de eucalipto à inoculação de duas espécies de Trichoderma, por diferentes métodos de inoculação. Em todas as variáveis analisadas não foram verificadas diferenças significativas em relação às espécies de Trichoderma $(p>0,05)$, bem como entre os métodos de inoculação utilizados $(p>0,05)$. Também não foram verificadas interações significativas entre esses fatores. Entretanto, ao se comparar a testemunha (sem Trichoderma) com os tratamentos que compõem o fatorial (com Trichoderma), verificaram-se diferenças significativas para as variáveis $\mathrm{D}(p=0,0203), \mathrm{NF}(p=0,0279), \mathrm{MSA}(p=$ 
0,0073), MST $(p=0,0115), \mathrm{H} / \mathrm{MSA}(p=0,0016)$ e IQD $(p=0,0109)$, sendo os resultados favoráveis ao maior desenvolvimento e qualidade das mudas obtidos quando se usou o Trichoderma. Já para $\mathrm{H}$ $(p=0,1631), \operatorname{MSR}(p=0,0609)$ e H/D $(p=0,2669)$ não se verificou diferenças entre os tratamentos.

Tabela 1. Resumo da análise de variância das variáveis analisadas em mudas clonais de Eucalyptus camaldulensis Dehnh. (clone 58), 95 dias após o estaqueamento.

Table 1. Summary of analysis of variance for variables analyzed in clonal seedlings of Eucalyptus camaldulensis Dehnh. (clone 58), 95 days after staking.

\begin{tabular}{|c|c|c|c|c|c|c|c|c|c|c|}
\hline \multirow{2}{*}{ FV } & \multirow{2}{*}{ GL } & \multicolumn{9}{|c|}{ Quadrado Médio } \\
\hline & & $\mathbf{H}$ & D & NF & MSA & MSR & MST & $H / D$ & H/MSA & IQD \\
\hline$T$ & 1 & $2,17^{\text {ns }}$ & $0,02^{\text {ns }}$ & $0,06^{\mathrm{ns}}$ & $0,01^{\text {ns }}$ & 0,00 ns & $0,01^{\text {ns }}$ & $0,00^{\mathrm{ns}}$ & $0,04^{\mathrm{ns}}$ & $0,000 \mathrm{~ns}$ \\
\hline MI & 3 & 1,69 ns & $0,05^{\text {ns }}$ & 6,39 ns & 0,03 ns & 0,01 ns & 0,06 ns & 0,47 ns & $63,63^{n s}$ & 0,000 ns \\
\hline $\mathrm{T} \times \mathrm{Ml}$ & 3 & 3,99 ns & $0,011^{\mathrm{ns}}$ & 1,22 ns & $0,03^{\text {ns }}$ & 0,01 ns & $0,05^{\mathrm{ns}}$ & $0,48^{\mathrm{ns}}$ & $62,72^{\text {ns }}$ & $0,000 \mathrm{~ns}$ \\
\hline Cont. x Fat. & 1 & 7,49 ns & 0,16 * & 18,06 * & 0,10 ** & 0,02 ns & 0,23 * & 0,68 ns & 333,75 ** & 0,002 * \\
\hline Resíduo & 24 & 3,62 & 0,02 & 2,51 & 0,01 & 0,01 & 0,03 & 0,52 & 26,53 & 0,000 \\
\hline CV (\%) & & 7,82 & 6,43 & 20,24 & 15,74 & 26,58 & 17,12 & 6,51 & 13,07 & 18,24 \\
\hline Média Geral & & 24,34 & 2,20 & 7,83 & 0,64 & 0,38 & 1,01 & 11,08 & 39,42 & 0,08 \\
\hline Média Fatorial & & 24,50 & 2,22 & 8,19 & 0,65 & 0,39 & 1,04 & 11,03 & 38,34 & 0,08 \\
\hline Média Control & & 23,05 & 2,01 & 5,00 & 0,48 & 0,30 & 0,79 & 11,47 & 48,03 & 0,06 \\
\hline
\end{tabular}

$\mathrm{T}=$ Trichoderma; $\mathrm{MI}=$ método de inoculação; Cont. = tratamento controle (testemunha); Fat. = fatorial; CV (\%) = coeficiente de variação experimental; $\mathrm{FV}=$ fonte de variação; $\mathrm{GL}=$ graus de liberdade; $\mathrm{H}=$ altura da parte aérea $(\mathrm{cm}) ; \mathrm{D}=$ diâmetro do coleto $(\mathrm{mm}) ; \mathrm{NF}=$ número de folhas; $M S A=$ massa seca aérea $(\mathrm{g}) ; \mathrm{MSR}=$ massa seca das raízes (g); MST = massa seca total ( $\mathrm{g}$ ); H/D = relação altura/diâmetro; H/MSA = relação altura/massa seca aérea; IQD = índice de qualidade de Dickson; ns = não significativo; * = significativo a $5 \%$ de probabilidade de erro; ** = significativo a $1 \%$ de probabilidade de erro.

Quando avaliada a diferença relativa entre os tratamentos com e sem Trichoderma, verifica-se, de forma geral, que o uso do Trichoderma proporcionou melhor desempenho para todas as variáveis analisadas (Figura 1), com incrementos variando de 1,8\% a 13,0\% para $\mathrm{H}, 4,3 \%$ a $16,5 \%$ para D, $40,0 \%$ a $110,0 \%$ para NF, $21,9 \%$ a $70,4 \%$ para MSA, $14,5 \%$ a $54,7 \%$ para MSA, $19,1 \%$ a $64,8 \%$ para MST, $-6,1 \%$ a $1,3 \%$ para a relação $H / D,-35,2 \%$ a $-10,9 \%$ para a relação $H / M S A$ e $24,2 \%$ a $70,4 \%$ para IQD. Dentre os tratamentos avaliados, o T. virens, inoculado via tratamento das miniestacas (TM), tendeu a apresentar maior superioridade em relação aos demais, proporcionando os melhores resultados para a maioria das variáveis analisadas, exceto para H e relação H/D.

Os resultados deste trabalho corroboram com os encontrados por outros autores para espécies de eucalipto. Carvalho Filho et al. (2008a), ao avaliarem a ação de Trichoderma harzianum (100 mL da suspensão de esporos $-10^{7}$ conídios $\mathrm{mL}^{-1}$ - para $30 \mathrm{Kg}$ de substrato) na promoção do crescimento de mudas via sementes (E. camaldulensis) e miniestaquia (E. urophylla x E. grandis - clone GG100), verificaram incrementos superiores a $43 \%$ para $\mathrm{H}, 114 \%$ para MSA e $37,5 \%$ para MSR.

Santos et al. (2008) verificaram incrementos de até 79,5\% na MSR e 54,4\% na MSA em mudas do clone GG100 submetidas à isolados de T. harzianum $\left(100 \mathrm{~mL}\right.$ da suspensão de esporos - $10^{7}$ conídios $\mathrm{mL}^{-1}$ - para $30 \mathrm{Kg}$ de substrato, acrescida de três pulverizações foliares). Entretanto, cabe ressaltar que estes autores verificaram que o isolado CEN512 (T. atroviride) reduziu em cerca de 17\% a massa seca das mudas. Por sua vez, Fortes et al. (2007), ao utilizar um isolado de Trichoderma sp. (E15) inoculado ao substrato através de grãos de arroz colonizados pelo fungo, obtiveram aumento significativo no enraizamento de microestacas de Eucalyptus sp., com incremento de 33,48\% na taxa de enraizamento, quando comparada com a testemunha.

Além das espécies de eucalipto, o Trichoderma também tem apresentado resultados satisfatórios para outras espécies florestais. Machado et al. (2015) verificaram que isolados de T. harzianum, inoculados via pó de arroz colonizado incorporado ao substrato, não interferiram na emergência das plântulas, porém apresentaram potencial como promotores de crescimento de mudas de cambará (Gochnatia polymorpha). Donoso et al. (2008) observaram que a presença de T. harzianum (produto comercial), inoculado via fertirrigação, quando associado com substrato a base de composto orgânico, permitiu um incremento significativo em altura e biomassa, bem como no desenvolvimento do sistema radicular de mudas de Pinus radiata. Já em mudas de Hevea brasiliensis, Promwee et al. (2014) verificaram que a incorporação de T. harzianum ao substrato proporcionou maior crescimento em mudas cultivadas em vasos, além de ter incrementado o conteúdo de fósforo nas folhas. 


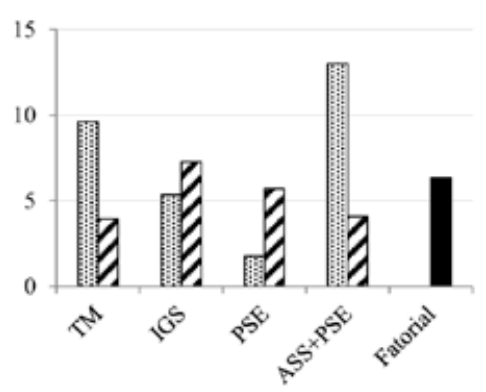

MSA

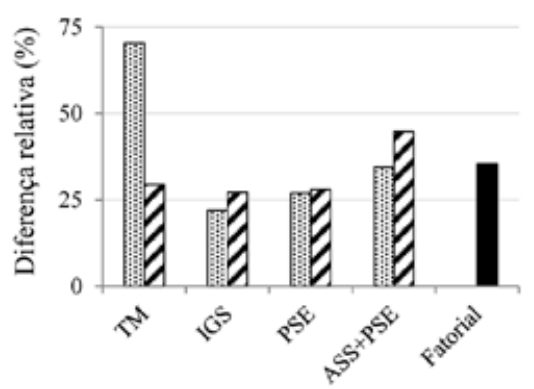

H/D

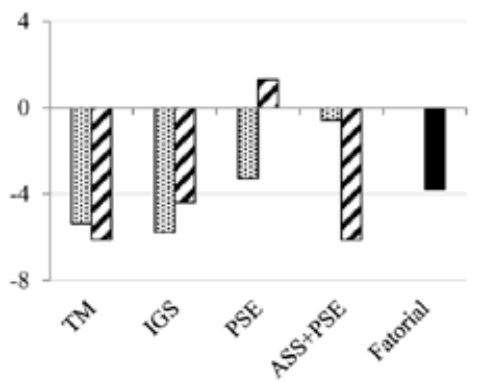

D

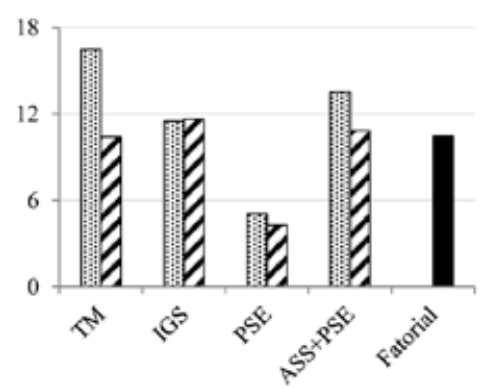

MSR

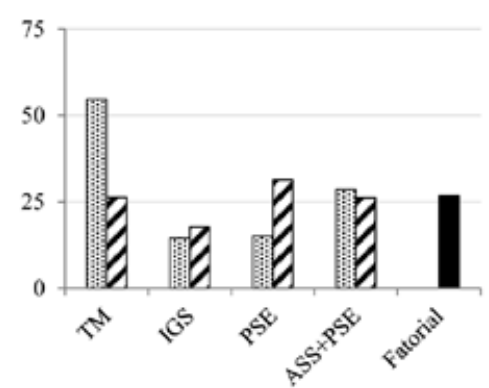

H/MSA

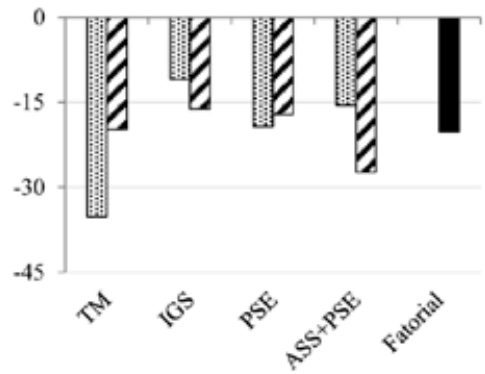

Métodos de inoculação do Trichoderma

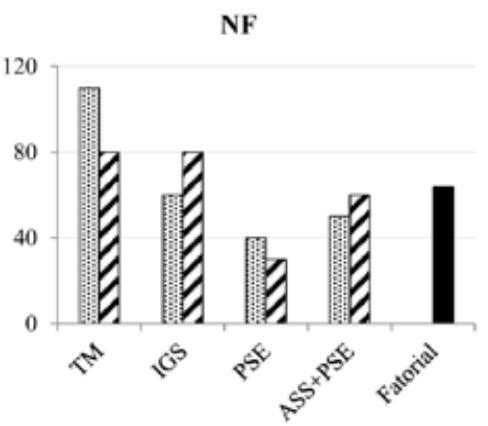

MST

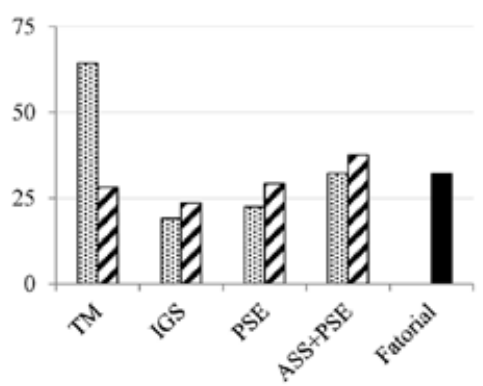

IQD

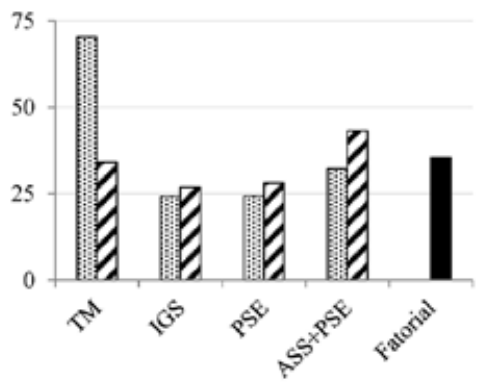

B $T$. virens $\square T$. harziamum $\mathbf{a}$ Fatorial

Figura 1. Diferença relativa entre as médias dos tratamentos e a testemunha (sem Trichoderma) em mudas clonais de Eucalyptus camaldulensis Dehnh. (clone 58). Em que: $\mathrm{H}=$ altura da parte aérea, $\mathrm{D}=$ diâmetro do coleto; $\mathrm{NF}=$ número de folhas; $\mathrm{MSA}=$ massa seca aérea; $\mathrm{MSR}=$ massa seca das raízes; $\mathrm{MST}=$ massa seca total; H/D = relação altura/diâmetro; H/MSA = relação altura/massa seca aérea; IQD = índice de qualidade de Dickson; TM = tratamento das miniestacas; IGS = incorporação de grãos de arroz colonizados ao substrato; PSE = pulverização de suspensão de esporos; ASS+PSE = adição de suspensão de esporos ao substrato + pulverização de suspensão de esporos.

Figure 1. Relative difference between the means of the treatments and control (without Trichoderma) in clonal seedlings of Eucalyptus camaldulensis Dehnh. (clone 58). Where: $\mathrm{H}=$ shoot height, $\mathrm{D}=$ stem diameter; $\mathrm{NF}$ $=$ number of leaves; $M S A=$ shoot dry mass; MSR = root dry mass; $M S T=$ total dry mass; $H / D=$ height/ diameter; $\mathrm{H} / \mathrm{MSA}=$ height/shoot dry mass; IQD = Dickson quality index; $\mathrm{TM}=$ treatment of mini-cutting; IGS = incorporating grains of rice colonized in the substrate; PSE = spraying of spore suspension; ASS+PSE $=$ adding spore suspension in the substrate + spraying of spore suspension.

Os resultados do presente trabalho, juntamente com os dos autores apresentados anteriormente, demonstram que, independente do método de inoculação, o Trichoderma apresenta potencial para ser utilizado na promoção do desenvolvimento de mudas. Segundo Brotman et al. (2010), espécies de Trichoderma podem promover aumentos de até 300\% no crescimento de plantas. Para Harman et al. (2004), a interferência desse fungo no desenvolvimento de plantas e no aumento de produtividade, ocorre devido a sua capacidade em colonizar as raízes.

O teste de colonização endofítica revelou que T. virens e T. harzianum são capazes de colonizar as raízes de mudas de eucalipto, não tendo sido detectados no caule e nas folhas (Tabela 2). Carvalho Filho et al. (2008a), também verificaram a colonização de dois isolados de T. harzianum e um de T. asperellum apenas nas raízes de mudas de eucalipto (clone GG100). Entretanto, alguns estudos demonstram que o Trichoderma também é capaz de colonizar a parte aérea de plantas, como Theobroma cacao (BAILEY et al., 2008; 2009; EVANS et al., 2003), Hevea spp. (CHAVERRI et al., 2011) e Strychnos cogens (SOUZA et al., 2004). 
Tabela 2. Presença (+) e ausência (-) de Trichoderma spp. em diferentes partes de mudas clonais de Eucalyptus camaldulensis Dehnh. (clone 58), detectadas pelo teste de colonização endofítica.

Table 2. Presence (+) and absence (-) of Trichoderma spp. in different parts of clonal seedlings of Eucalyptus camaldulensis Dehnh. (clone 58), detected by endophytic colonization test.

\begin{tabular}{llccc}
\hline Espécie de Trichoderma & Método de inoculação & Raiz & Caule & Folha \\
\hline T. virens & TM & + & - & - \\
T. virens & IGS & + & - & - \\
T. virens & PSE & - & - & - \\
T. virens & ASS+PSE & - & - & - \\
T. harzianum & TM & - & - & - \\
T. harzianum & IGS & + & - & - \\
T. harzianum & PSE & - & - & - \\
T. harzianum & ASS+PSE & + & - & - \\
Tratamento Controle & & - & - & - \\
\hline
\end{tabular}

TM = tratamento das miniestacas; IGS = incorporação de grãos de arroz colonizados ao substrato; PSE = pulverização de suspensão de esporos; ASS +PSE = adição de suspensão de esporos ao substrato + pulverização de suspensão de esporos.

A interação Trichoderma-planta geralmente ocorre na região das raízes, podendo ser em diferentes níveis. Segundo Metcalf e Wilson (2001), algumas linhagens colonizam apenas regiões específicas das raízes, enquanto outras, denominadas de rizosfera-competentes, colonizam toda a superfície radicular, penetram no espaço intercelular das primeiras camadas da epiderme e permanecem em associação com as raízes por longos períodos. Para Harman (2000), as linhagens mais eficazes no controle biológico e promoção de crescimento são aquelas capazes de estabelecer interações duradouras com a planta, pois seus efeitos perduram por todo ou grande parte do ciclo de vida da planta.

O uso do Trichoderma mostrou ser uma alternativa promissora a ser adotada no processo produtivo dos viveiros florestais, uma vez que o mesmo é capaz de promover o desenvolvimento de mudas, como constatado no presente trabalho, apresentando ainda a vantagem de realizar o biocontrole de fungos fitopatogênicos, como constatado por Mafia et al. (2003), Carvalho Filho et al. (2008b), Zaldúa e Sanfuentes (2010) e Maciel et al. (2012) em mudas de eucalipto. Dessa forma, o Trichoderma pode contribuir para a redução do consumo de insumos como fungicidas e adubos nos viveiros florestais, contribuindo para a maior sustentabilidade dessa atividade.

Contudo, o uso do Trichoderma deve ser utilizado com critério, uma vez que, segundo Benítez et al. (2004) e Stewart e Hill (2014), a promoção do desenvolvimento pode ser altamente variável devido a vários fatores limitantes, incluindo tipo de cultura, condições de desenvolvimento, taxa de inóculo e tipo de formulação. Portanto, torna-se importante a realização de novos estudos para avaliar os efeitos do Trichoderma em diferentes espécies florestais e condições de manejo.

\section{CONCLUSÕES}

Trichoderma virens e Trichoderma harzianum apresentam eficiência como promotores de crescimento de mudas clonais de Eucalyptus camaldulensis em condições de viveiro.

O tratamento das minestacas, pela imersão da base em pó de arroz colonizado com Trichoderma virens, mostra ser o tratamento mais eficiente na promoção do desenvolvimento e qualidade das mudas de eucalipto.

As duas espécies de Trichoderma são capazes de colonizar endofiticamente as raízes de mudas do clone avaliado.

\section{REFERÊNCIAS BIBLIOGRÁFICAS}

AZARMI, R.; HAJIEGHRARI, B.; GIGLOU, A. Effect of Trichoderma isolates on tomato seedling growth response and nutrient uptake. African Journal of Biotechnology, Lagos, v. 10, n. 31, p. 5850-5855, 2011.

BAILEY, B. A., STREM M. D.; WOOD, D. Trichoderma species form endophytic associations within Theobroma cacao trichomes. Mycological Research, Cambridge, v. 113, n. 12, p.1365-1376, 2009. 
Azevedo et al. - Efeito de Trichoderma spp. no

crescimento de mudas clonais de Eucalyptus camaldulensis

BAILEY, B. A.; BAE, H.; STREM, M. D.; CROZIER, J.; THOMAS, S. E.; SAMUELS, G. J.; VINYARD, B. T.; HOLMES, K. A. Antibiosis, mycoparasitism, and colonization success for endophytic Trichoderma isolates with biological control potential in Theobroma cacao. Biological Control, Orlando, v. 46, n. 1, p.24-35, 2008.

BENÍTEZ, T.; RINCÓN, A. M.; LIMÓN, M. C.; CODÓN, A. C. Bioncontrol mechanisms of Trichoderma strains. International Microbiology, Barcelona, v. 7, n. 4, p. 249-260, 2004.

BÖHM, W. Methods of studying root systems. Berlin: Springer-Verlag, 1979. 188 p.

BROTMAN, Y.; KAPUGANTI, J. G.; VITERBO, A. Trichoderma. Current Biology, Cambridge, v. 20, n. 9, p. R390-R391, 2010.

BROTMAN, Y.; LANDAU, U.; CUADROS-INOSTROZA, Á.; TAKAYUKI, T.; FERNIE, A. R.; CHET, I.; VITERBO, A.; WILLMITZER, L. Trichoderma-plant root colonization: escaping early plant defense responses and activation of the antioxidant machinery for saline stress tolerance. PLoS Pathogens, San Francisco, v. 9, n. 3, e1003221, 2013.

CARVAlHO FILHO, M. R.; MEllO, S. C. M.; SANTOS, R. P.; MENÊZES, J. E. Avaliação de isolados de Trichoderma na promoção de crescimento, produção de ácido indolacético in vitro e colonização endofítica de mudas de eucalipto. Brasília: Embrapa Recursos Genéticos e Biotecnologia, 2008a. 16 p. (Boletim de Pesquisa e Desenvolvimento, 226).

CARVAlHO FILHO, M. R.; MENÊZES, J. E.; MELLO, S. C. M.; SANTOS, R. P. Avaliação de isolados de Trichoderma no controle da mancha foliar do eucalipto in vitro e quanto à esporulação em dois substratos sólidos. Brasília: Embrapa Recursos Genéticos e Biotecnologia, 2008b. 21 p. (Boletim de Pesquisa e Desenvolvimento, 225).

CHAGAS JÚNIOR, A. F.; OliveIRA, A. G.; SANTOS, G. R.; REIS, A. F. B.; CHAGAS, L. F. B. Promoção de crescimento em feijão-caupi inoculado com rizóbio e Tricoderma spp. no cerrado. Revista Caatinga, Mossoró, v. 27, n. 3, p.190-199, 2014.

CHAVERRI, P.; GAZIS, R. O.; SAMUELS, G. J. Trichoderma amazonicum, a new endophytic species on Hevea brasiliensis and H. guianensis from the Amazon basin. Mycologia, Lawrence, v. 103, n. 1, p. 139-151, 2011.

CHÁVEZ, D.; PEREIRA, G.; MACHUCA, A. Estimulación del crecimiento en plántulas de Pinus radiata utilizando hongos ectomicorrícicos y saprobios como biofertilizantes. Bosque, Valdívia, v. 35, n. 1 p. 57-63, 2014.

DONOSO, E.; LOBOS, G.; ROJAS, N. Efecto de Trichoderma harzianum y compost sobre el crecimiento de plántulas de Pinus radiata en vivero. Bosque, Valdívia, v. 29, n. 1, p. 52-57, 2008.

EVANS, H. C.; HOLMS, K. A.; THOMAS, S. E. Endophytes and mycoparasites associated whit an indigenous forest tree, Theobromae gibereli, in Ecuador and a preliminary assessment of their potential as biocontrol agents of cocoa diseases. Mycological Progress, Heidelberg, v. 2, p. 149-160, 2003.

FERREIRA, E. B.; CAVALCANTI, P. P.; NOGUEIRA, D. A. ExpDes: An R Package for ANOVA and Experimental Designs. Applied Mathematics, New York, v. 5, n. 19, p. 2952-2958, 2014.

FORTES, F. O.; SILVA, A. C. F.; ALMANÇA, M. A. K.; TEDESCO, S. B. Promoção de enraizamento de microestacas de um clone de Eucalyptus sp. por Trichoderma spp. Revista Árvore, Viçosa, v. 31, n. 2, p. 221-228, 2007.

GOMES, J. M.; PAIVA, H. P. Viveiros florestais (propagação sexuada). 3.ed, Viçosa: UFV, 2004. 116p (Caderno didático, 72). 
GRAVEL, V.; ANTOUN, H.; TWEDDELL, R. J. Growth stimulation and fruit yield improvement of greenhouse tomato plants by inoculation with Pseudomonas putida or Trichoderma atroviride: possible role of indole acetic acid (IAA). Soil Biology \& Biochemistry, Elmsford, v. 39, n. 8, p. 1968-1977, 2007.

HARMAN, G. E. Overview of mechanisms and of Trichoderma spp. Phytopathology, Saint Paul, v. 96, n. 2, p. 190-194, 2006.

HARMAN, G. E. Myth and dogmas of biocontrol changes in perceptions derived from research on Trichoderma harzianum T-22. Plant Disease, Saint Paul, v. 84, n. 4, p. 377-393, 2000.

HARMAN, G. E. HOWELL, C. R.; VITEBERBO, A.; CHET, I.; LORITO, M. Trichoderma species - opportunistic, avirulent plant syimbionts. Nature Reviews Microbiology, London, v. 2, p .43-56, 2004.

HERMOSA, R.; VITERBO, A.; CHET, I.; MONTE, E. Plant-beneficial effects of Trichoderma and of its genes. Microbiology, New York, v. 158, p. 17-25, 2012

MACHADO, D. F. M.; TAVARES, A. P.; LOPES, S. J.; SILVA, A. C. F. Trichoderma spp. na emergência e crescimento de mudas de cambará (Gochnatia polymorpha (Less.) Cabrera). Revista Árvore, Viçosa, v. 39, n. 1, p. 167-176, 2015.

MACHADO, D. F. M.; PARZIANELLO, F. R.; SILVA, A. C. F. e ANTONIOLLI, Z. I. Trichoderma no Brasil: o fungo e o bioagente. Revista de Ciências Agrárias, Lisboa, v. 35, n. 1, p. 274-288, 2012.

MACIEL, C. G.; LAZAROTTO, M. MEZZOMO, R.; POLETTO, I.; MUNIZ, M. F. B.; LIPPERT, D. B. Trichoderma spp. no biocontrole de Cylindrocladium candelabrum em mudas de Eucalyptus saligna. Revista Árvore, Viçosa, v. 36, n. 5, p. 825-832, 2012.

MAFIA, R. G.; ALFENAS, A. C.; MAFFIA, L. A.; VENTURA, G. M.; SANFUENTES, E. A. Encapsulamento de Trichoderma inhamatum para o controle biológico de Rhizoctonia solani na propagação clonal de Eucalyptus. Fitopatologia Brasileira, Brasília, v. 28, n. 1, p. 101-105, 2003.

METCALF, D. A.; WILSON, C. R, T. The process of antagonism of Sclerotium cepivorum in white rot affected onion roots by Trichoderma koningii. Plant Pathology, Malden, v. 50, p. 249-257, 2001.

PEDRO, E. A. S.; HARAKAVA, R.; LUCON, C. M. M.; GUZZO, S. D. Promoção do crescimento do feijoeiro e controle da antracnose por Trichoderma spp. Pesquisa Agropecuária Brasileira, Brasília, v. 47, n. 11, p. 15891595, 2012.

PROMWEE, A.; ISSARAKRAISILA, M.; CHAMSWARNG, C.; YENJIT, P. Phosphate solubilization and growth promotion of rubber tree (Hevea brasiliensis Muell. Arg.) by Trichoderma Strains. Journal of Agricultural Science, Cambridge, v. 6, n. 9, p. 8-20, 2014.

RESENDE, M. L. OLIVEIRA, J. A.; GUIMARÃES, R. M.; PINHO, R. G. V.; VIEIRA, A. R. Inoculação de sementes de milho utilizando o Trichoderma harzianum como promotor de crescimento. Ciência e Agrotecnologia, Lavras, v. 28, n. 4, p. 793-798, 2004.

SABA, H.; VIBHASH, V.; MANISHA, M.; PRASHANT, K. S.; FARHAM, H.; TAUSEFF, A. Trichoderma - a promising plant growth stimulator and biocontrol agent. Mycosphere, Guiyang, p. 524-531, 2012.

SANTOS, H. A.; MELLO, S. C. M.; PEIXOTO, J. R. Associação de isolados de Trichoderma spp. e ácido indol-3butírico (AIB) na promoção de enraizamento de estacas e crescimento de maracujazeiro. Bioscience Journal, Uberlândia, v. 26, n. 6, p. 966-972, 2010. 
Azevedo et al. - Efeito de Trichoderma spp. no

crescimento de mudas clonais de Eucalyptus camaldulensis

SANTOS, R. P.; CARVALHO FILHO, M. R.; MARTINS, I. Avaliação de isolados de Trichoderma spp. e Gliocladium virens na promoção de crescimento em mudas de eucalipto e na produção de ácido indolacético in vitro. Brasília: Embrapa Recursos Genéticos e Biotecnologia, 2008. 13 p. (Boletim de Pesquisa e Desenvolvimento, 232).

SFB - SERVIÇO FLORESTAL BRASILEIRO. As florestas plantadas. Disponível em: < http://www.florestal.gov. br/snif/recursos-florestais/as-florestas-plantadas >. Acesso em: 19 jan 2016.

SHANMUGAIAH, V.; BALASUBRAMANIAN, N.; GOMATHINAYAGAM, S.; MANOHARAN, P. T.; RAJENDRAN, A. Effect of single application of Trichoderma viride and Pseudomonas fluorescens on growth cotton plants. African Journal of Agricultural Research, Nairobi, v. 4, n. 11, p. 1220-1225, 2009.

SILVA, A. N.; AZEVEDO, G. B.; ROCHA SOBRINHO, G.G.; NOVAES, Q. S. Efeito de produtos químicos e de Trichoderma spp. no controle de Fusarium solani do maracujazeiro. Interciencia, Caracas, v. 39, n. 6, p. 398403, 2014.

SILVA, V. N.; GUZZO, S. D.; LUCON, C. M. M.; HARAKAVA, R. Promoção de crescimento e indução de resistência à antracnose por Trichoderma spp. em pepineiro. Pesquisa Agropecuária Brasileira, Brasília, v. 46, n. 12, p. 1609-1618, 2011.

SINGH, V.; SINGH, P. N.; YADAV, R. L.; AWASTHI, S. K.; JOSHI, B. B.; SINGH, R. K.; LAL, R. J.; DUTTAMAJUMDER, S. K. Increasing the efficacy of Trichoderma harzianum for nutrient uptake and control of red rot in sugarcane. Journal of Horticulture and Forestry, Warwick, v. 2, n. 4, p. 66-71, 2010.

SOUZA, A. Q. L.; SOUZA, A. D. L; ASTOLFI FILHO, S.; PINHEIRO, M. L. B.; SARQUIS, M. I. M.; PEREIRA, J. O. Atividade antimicrobiana de fungos endofíticos isolados de plantas tóxicas da amazônia: Palicourea longiflora (aubl.) rich e Strychnos cogens bentham. Acta Amazonica, Manaus, v. 34, n. 2, p.185-195, 2004.

SRIVASTAVA, M.; SHAHID, M.; PANDEY, S.; SINGH, A.; KUMAR, V.; GUPTA, S.; MAURYA, M. Trichoderma genome to genomics: a review. Data Mining in Genomics \& Proteomics, London, v. 5, n. 3, p. 10162, 2014.

STEWART, A.; HILL, R. Applications of Trichoderma in plant growth promotion. In: GUPTA, V. K.; SCHMOLL, M.; HERRERA-ESTRELLA, A.; UPADHYAY, R. S.; DRUZHININA, I.; TUOHY, M. G. Biotechnology and biology of Trichoderma. Boston: Elsevier USA, 2014. p. 415-428.

SEI - SUPERINTENDÊNCIA DE ESTUDOS ECONÔMICOS E SOCIAIS DA BAHIA. 2015. Disponível em: < http:// www.sei.ba.gov.br >. Acesso em: 20 jul 2015.

ZALDÚA, S.; SANFUENTES, E. Control of Botrytis cinerea in Eucalyptus globulus mini-cuttings using Clonostachys and Trichoderma strains. Chilean Journal of Agricultural Research, Chillán, v. 70, n .4, p. 576-582, 2010.

Recebido em 30/03/2016

Aceito para publicação em 12/01/2017 